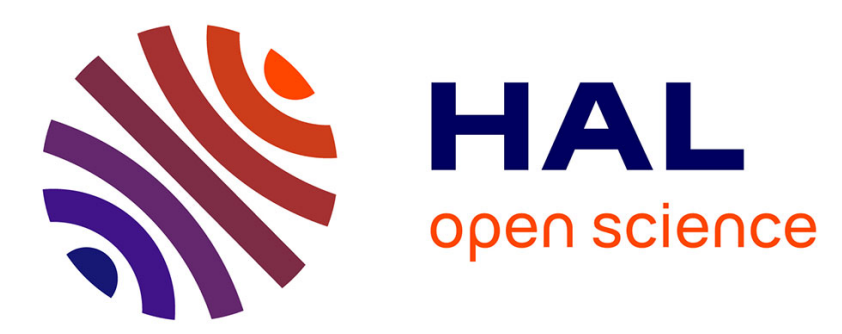

\title{
Un language et un serveur de transformation de graphes pour le Web de données
}

Olivier Corby, Catherine Faron Zucker

\section{To cite this version:}

Olivier Corby, Catherine Faron Zucker. Un language et un serveur de transformation de graphes pour le Web de données. Revue des Sciences et Technologies de l'Information - Série RIA : Revue d'Intelligence Artificielle, 2016, Numéro spécial Ingénierie des connaissances, 30 (5), pp.21. 10.3166/RIA.30.607627. hal-01330124

\author{
HAL Id: hal-01330124 \\ https://hal.inria.fr/hal-01330124
}

Submitted on 24 Dec 2021

HAL is a multi-disciplinary open access archive for the deposit and dissemination of scientific research documents, whether they are published or not. The documents may come from teaching and research institutions in France or abroad, or from public or private research centers.
L'archive ouverte pluridisciplinaire HAL, est destinée au dépôt et à la diffusion de documents scientifiques de niveau recherche, publiés ou non, émanant des établissements d'enseignement et de recherche français ou étrangers, des laboratoires publics ou privés. 


\title{
Un language et un serveur de transformation de graphes pour le Web de données
}

\author{
Olivier Corby ${ }^{1} \&$ Catherine Faron ${ }^{2}$ \\ (1) Inria Université Côte d'Azur, I3S, olivier.corby@inria.fr \\ (2) Université Côte d'Azur, I3S, Inria, faron@i3s.unice.fr
}

\begin{abstract}
In this paper we first present the STTL (SPARQL Template Transformation Language) language for the transformation of RDF graphs, designed as an extension of SPARQL. Then we present a STTL-based platform for the design of Web browsers for the Linked Data. This platform is a SPARQL endpoint augmented with an STTL engine and services to run STTL transformations. Finally, we show the capabilities of the platform by presenting three Web browsers: a first one to execute SPARQL queries and present teir results in HTML; a second one to navigate on the DBpedia RDF graph with HTML presentation formats adapted to certain types of data; a third one that generates a mashup of a local $\mathrm{RDF}$ graph with the DBpedia RDF graph and provides a unified view; and a last one to manage and exploit a base of predefined SPARQL queries.
\end{abstract}

\section{Résumé}

Dans cet article nous commençons par présenter le langage STTL (SPARQL Template Transformation Language) pour la transformation de graphes RDF, conçu comme une extension du langage SPARQL. Nous présentons ensuite une plate-forme qui implémente ce langage pour permettre la conception de navigateurs Web offrant une navigation hypertextuelle en HTML dans des graphes RDF sur le Web de données. La plateforme se présente sous la forme d'un serveur HTTP embarquant, outre un service SPARQL, un moteur de transformation STTL et des services Web permettant d'exécuter des transformations. Nous montrons les capacités du système en présentant quatre navigateurs : un premier pour exécuter des requêtes SPARQL sur un graphe RDF local ou sur le Web et pour présenter les résultats en HTML; un second pour naviguer sur le graphe RDF de DBpedia avec des formats de présentation dédiés à certains types de ressources; un troisième pour présenter une vue unifiée d'un graphe local lié au graphe de DBpedia; et un dernier pour gérer et exploiter une base de requêtes SPARQL prédéfinies. 


\section{Introduction}

Le Web de documents structurés qui repose sur le standard XML a rapidement été muni du standard XSLT pour engendrer des formats de présentation tels que HTML ou bien pour écrire des transformations de XML vers XML. De la même manière, le Web de données qui repose sur le standard RDF a maintenant besoin d'un langage de transformation pour présenter les données RDF dans des formats lisibles tels que HTML et pour écrire des transformations de RDF vers RDF.

La transformation et la présentation de données RDF est encore un problème ouvert. Nous avons proposé dans [3] et [5] un langage de règles de transformation pour RDF, SPARQL Template Transformation Language (STTL), dont le format de sortie est textuel - sans restriction a priori sur le format de texte. L'état de l'art sur les autres solutions existantes pour transformer des données RDF présenté dans les articles ci-dessus mentionnés montre qu'elles sont toutes liées à la syntaxe XML ou à un format spécifique de sortie, ou aux deux, sauf Fresnel [1]. Mais Fresnel s'intéresse à la présentation des données RDF et ne traite pas du problème plus général de leur transformation. STTL permet une approche générique pour écrire des transformations RDF vers différents formats de sortie.

Nous nous intéressons dans cet article plus particulièrement à la transformation de données RDF en HTML et nous présentons une plate-forme basée sur le langage STTL, nommée ALIGATOR (acronyme de A LInked data naviGATOR), et permettant de concevoir des navigateurs pour le Web des données liées et de présenter ces données en HTML. ALIGATOR est constitué d'un serveur HTTP, un service REST, un moteur de transformations STTL et une bibliothèque de transformations STTL. Le code HTML engendré par les transformations contient des liens hypertextes vers le serveur ce qui permet d'offrir une navigation hypertextuelle sur des graphes RDF.

Cet article est organisé comme suit : la section 2 présente brièvement le langage STTL, la section 3 présente le service REST appelant le moteur de transformations STTL. La section 4 présente différentes applications Web permettant de présenter et naviguer sur des données RDF.

\section{Le langage de transformation STTL}

Le langage STTL que nous avons conçu est une extension du langage $S P A R Q L$ 1.1 Query avec une clause TEMPLATE permettant d'engendrer un résultat sous forme de texte à partir des solutions d'une clause WHERE. La clause TEMPLATE peut contenir du texte, des variables et des expressions. Les variables sont remplacées par les valeurs trouvées dans les solutions, les expressions sont évaluées et le tout est concaténé sous forme d'une chaîne de caractères qui est le résultat retourné par la clause TEMPLATE. L'exemple suivant montre la traduction d'un énoncé OWL exprimé dans la syntaxe RDF de OWL - une restriction de type "allValuesFrom" - dans la syntaxe fonctionnelle de OWL. 


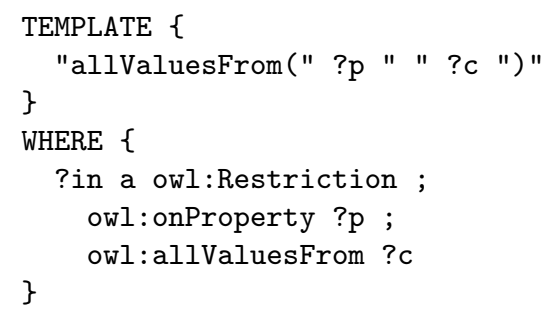

Une transformation STTL est un ensemble de tels patrons de transformation dédiés à la transformation d'énoncés RDF dans un certain modèle (e.g. les données de DBpedia sur les rois de France) ou pour un certain langage (e.g. OWL/RDF) dans un format textuel (e.g. une présentation des rois de France en HTML ou une représentation d'énoncés OWL dans la syntaxe fonctionnelle du langage). L'exécution d'un patron peut récursivement déclencher l'exécution d'autres patrons de transformation ; l'appel à un patron se fait au moyen d'une fonction d'extension st:apply-templates ${ }^{1}$ appelée dans la clause TEMPLATE. Une variante de l'exemple précédent est le patron suivant :

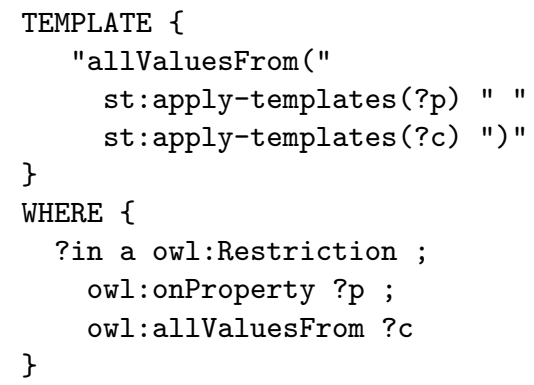

Dans ce patron de transformation, l'appel de la fonction st:apply-templates avec la variable ?p en paramètre déclenchera la sélection d'un autre patron de transformation qui sera appliqué sur le résultat (binding) associé à la variable ?p lors de l'appariement du graphe requête de la clause WHERE avec le graphe RDF à transformer. La même chose se produira pour la variable ?c. Des patrons nommés sont également prédéfinis (et d'autres peuvent être définis et nommés par le programmeur STTL) qui peuvent être exécutés directement par un appel à la fonction d'extension st : call-template. Voici un patron de transformation équivalent au précédent où les patrons appelés dans la clause TEMPLATE sont indiqués explicitement : le patron st:property dédié à la présentation d'une propriété dans la syntaxe fonctionnelle de OWL est appelé avec la variable ?p en paramètre et le patron st : value dédié à la présentation des valeurs de propriété est appelé avec la variable ?c en paramètre.

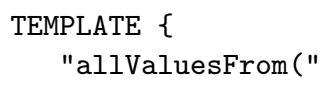




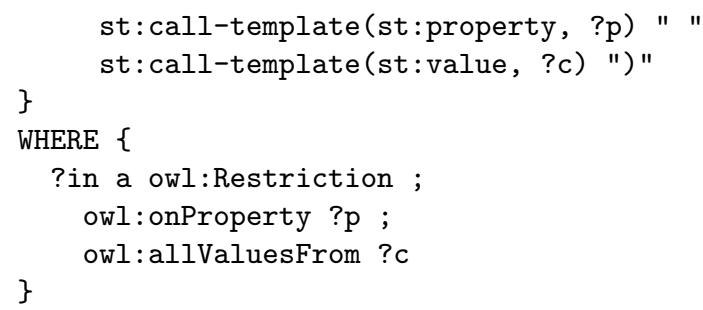

\subsection{Syntaxe de STTL}

Voici la syntaxe de STTL dans la syntaxe de SPARQL 1.1 Query Language [7]. STTL est ainsi vu comme une extension de SPARQL avec la clause Template - Prologue définit les préfixes, PrimaryExpression est une constante, une variable, un appel de fonction ou une expression parenthésée.

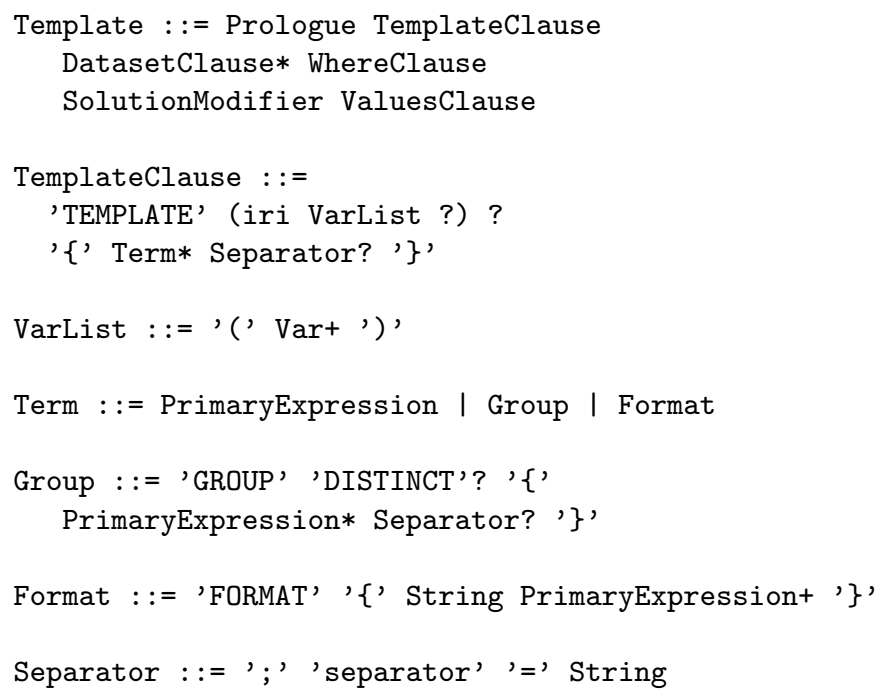

La table ci-dessous présente les fonctions principales de STTL.

\begin{tabular}{|l|l|}
\hline Name & Description \\
\hline st:apply-templates & Applique la transformation courante sur le focus \\
\hline st:apply-templates-with & Applique une transformation sur le focus \\
\hline st:apply-templates-all & Applique tous les templates sur le focus \\
\hline st:call-template & Applique un template nommé sur le focus \\
\hline st:call-template-with & Applique un template nommé d'une transformation sur le focus \\
\hline st:process & Définit le traitement des variables \\
\hline st:get & Trouve une propriété du contexte \\
\hline st:set & Assigne une propriété au contexte \\
\hline st:turtle & Retourne un terme en syntaxe Turtle \\
\hline
\end{tabular}

Le schema de traduction suivant décrit comment les expressions du langages sont traduites en SPARQL. La règle (1) traduit une clause TEMPLATE en une 
Soit $\Omega$ la séquence de solutions résultant de l'évaluation de la requête SPARQL issue de la compilation d'un patron STTL. Le patron échoue si la séquence est vide. Si elle n'est pas vide, le résultat du patron est le résultat de l'opération d'aggrégation de l'algèbre de SPARQL ${ }^{3}$ group_concat suivant :

Aggregation((?out), group_concat, scalarvals, $\{1 \rightarrow \Omega\}$ )

où scalarvals correspond à l'argument sep de la clause TEMPLATE.

\subsection{Traitement par défaut}

Par défaut, la fonction st:process retourne le terme RDF en argument dans la syntaxe Turtle. Il est possible de redéfinir le comportement de cette fonction pour, par exemple, exécuter la fonction st:apply-templates. Pour cela, on dispose du language FunSPARQL de définition de fonctions directement en SPARQL [4]. L'exemple ci-dessous montre une redéfinition de la fonction st:process qui appelle le moteur de transformation quand l'argument est un nœud vide (blank node) et qui retourne le format Turtle sinon.

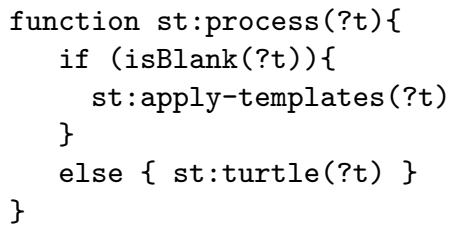

\subsection{Comparaison de STTL et XSLT}

STTL et XSLT sont relativement similaires dans leurs fonctionnalités et leur expressivité. Toutefois, XSLT traite des arbres XML où les arcs sont ordonnés alors que STTL traite des graphes RDF dans lesquels les arcs ne sont pas ordonnés. Ainsi, une requête XSLT/XPath qui recherche le 3ème fils d'un sommet n'a pas d'équivalent direct avec STTL mais cela peut être simulé avec des listes ou des conteneurs.

Les deux langages sont basés sur des règles de transformation déclaratives et possèdent les opérations apply-templates et call-template. Les deux langages possèdent une instruction conditionnelle, le groupement et le tri des solutions. XSLT possède la répétition explicite xsl:for-each alors qu'avec STTL celle-ci est implicite : la clause TEMPLATE s'applique à toutes les solutions.

Un template XSLT peut traiter plusieurs patrons successifs tandis que STTL ne possède qu'une seule clause WHERE. STTL hérite de tous les énoncés SPARQL 1.1, y compris les chemins de propriétés (property paths) et les services qui permettent d'interroger des graphes distants selon les principes du Linked Data.

\footnotetext{
${ }^{3}$ http://www.w3.org/TR/sparql11-query/\#aggregateAlgebra
} 


\section{Le serveur de transformation}

Nous présentons dans cette partie la plate-forme ALIGATOR qui repose sur le langage STTL et permet de réaliser des navigateurs hypertextes pour le Web de données.

Plus précisément, le système repose sur un serveur HTTP Jetty avec des services Web REST qui implémentent le protocole SPARQL 1.1 ${ }^{4}$. Un SPARQL endpoint exécute des requêtes SPARQL convoyées par HTTP et retourne les résultats également par HTTP. Ces résultats sont exprimés dans les formats standards SPARQL Query Results XML Format, Turtle, RDF/XML ou JSONLD.

Outre cette implémentation standard, ALIGATOR fournit un service Web qui permet d'exécuter des transformations STTL pour engendrer du code HTML à partir de données RDF. Nous appelons RDF2HTML ces transformations particulières. Ce service permet de réaliser des navigateurs hypertextes sur un graphe RDF local ou distant.

\subsection{Le service STTL}

Le service STTL répond à deux scénarios décrits sur la Figure 1. Dans le Scénario 1, une transformation RDF2HTML est exécutée sur un graphe RDF. Etant donné un URI, la transformation engendre une page HTML décrivant la ressource RDF correspondante. Le code HTML contient des liens hypertextes, vers le service STTL, pour décrire les ressources liées à cet URI. Cliquer sur ces liens hypertextes déclenche un appel au service STTL qui applique la transformation STTL et engendre en retour une nouvelle page HTML. On implémente ainsi une navigation hypertexte sur un graphe RDF. La transformation peut, par exemple, engendrer une table HTML avec les triplets dont l'URI est le sujet ou l'objet, comme le fait DBpedia ${ }^{5}$.

Dans le Scénario 2, le service STTL exécute une requête SPARQL sur le graphe puis applique une transformation RDF2HTML sur le résultat de la requête. Pour les requêtes de la forme CONSTRUCT ou DESCRIBE, la transformation est exécutée directement sur le graphe RDF résultat de la requête. Pour les requêtes de la forme SELECT ou ASK, le résultat de la requête est traduit en graphe RDF en utilisant le vocabulaire publié par le W3C, RDF Data Access Working Group ${ }^{6}$. La transformation STTL est alors appliquée sur le graphe RDF ainsi produit.

Une requête pour une transformation STTL est envoyée à un service ALIGATOR dans un URL dont la partie hiérarchique se termine par /template et dont la partie requête contient des paires clé-valeur spécifiant la requête au serveur. La clé query, reprise du protocole SPARQL, permet d'indiquer une requête SPARQL à exécuter. La clé transform permet de spécifier l'URL de la transformation à appliquer sur le résultat de la requête ou sur le graphe du

\footnotetext{
${ }^{4}$ http://www.w3.org/TR/sparql11-protocol/

${ }^{5}$ e.g. http://dbpedia.org/resource/Berlin

${ }^{6}$ http://www.w3.org/2001/sw/DataAccess/tests/result-set.n3
} 

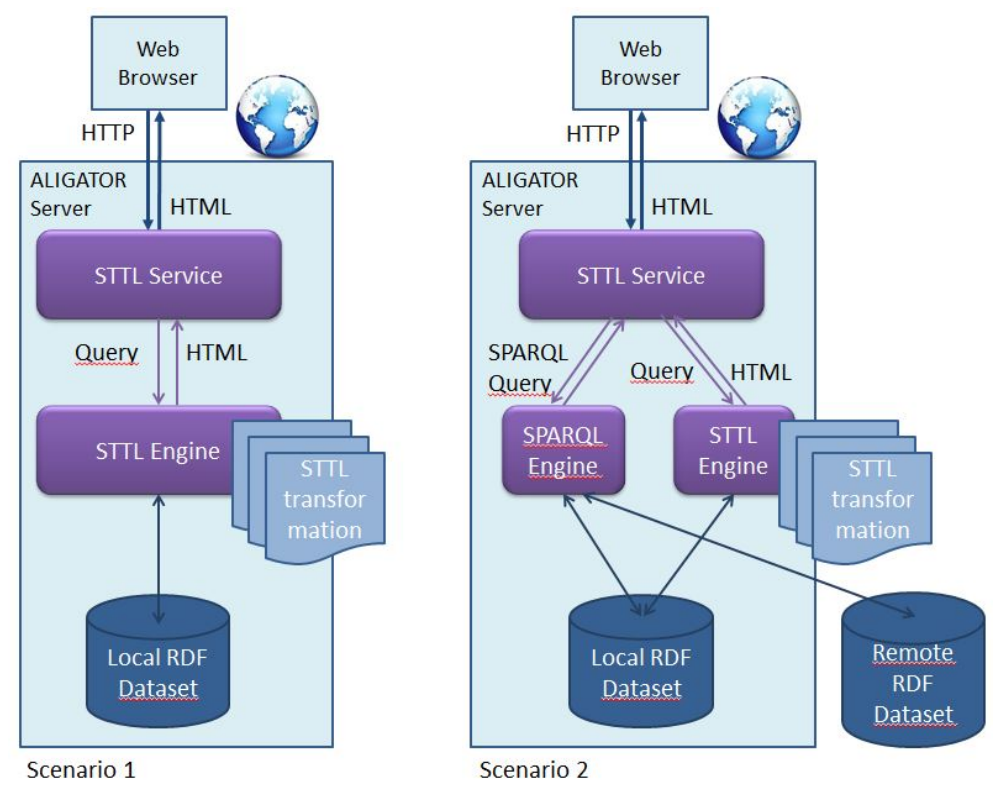

Figure 1: Architecture

serveur. Par exemple, l'URL suivant demande l'exécution d'une transformation STTL spécifiée par st:sparql sur le résultat d'une requête SPARQL qui retourne tous les triplets d'un graphe RDF donné.

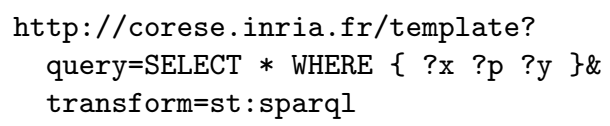

\subsection{Profil de transformation}

Un URL peut donc comporter une requête, une transformation ou les deux. Pour simplifier l'interaction avec le service STTL, nous introduisons la notion de profil de transformation RDF2HTML. Un profil permet de définir une chaîne de traitements simple, composée d'une requête SPARQL optionnelle et d'une transformation STTL, et de nommer ce traitement avec un URI. Un profil est décrit sous forme d'énoncés RDF : la classe st:Profile caractérise un profil, la propriété st:query définit un chemin (un URL) vers une requête SPARQL et la propriété st:transform spécifie l'URL d'une transformation STTL. Voici un exemple de profil :

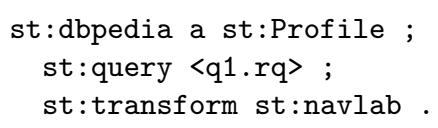

Dans cette description, q1.rq contient par exemple la requête SPARQL suivante qui interroge le serveur DBpedia : 
CONSTRUCT $\{? \mathrm{x}$ ?p ?y $\}$

WHERE $\{$ service <http://fr.dbpedia.org/sparql> $\{$ ? $\mathrm{x}$ ?p ?y $\}\}$

Dans l'URL transportant une requête pour une transformation STTL, l'URI d'un profil est indiqué par un argument profile dont la valeur est l'URI du profil :

http://corese.inria.fr/template?profile=st: dbpedia

Une requête peut préciser l'URI d'une ressource sur lequel focaliser l'exécution du service :

http://corese.inria.fr/template?profile=st: dbpedia

\&uri=http://fr.dbpedia.org/resource/Antibes

La requête SPARQL exécutée par le serveur peut être complétée par une clause de la forme st : get (st:uri) qui permet de récupérer dans le contexte d'exécution l'URI donné comme valeur de l'argument uri.

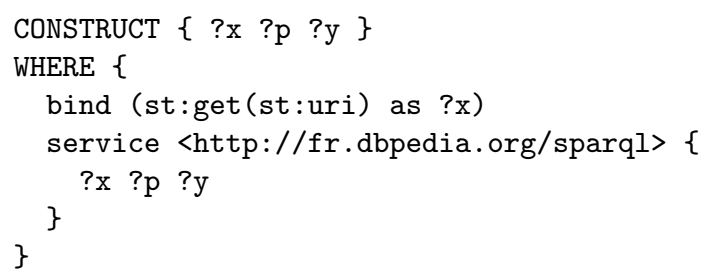

La définition d'un profil peut contenir une requête interrogeant un serveur distant, e.g. DBpedia, sur une ressource particulière, e.g. la ville d'Antibes, et peut appliquer au graphe résultat une transformation RDF2HTML qui engendre une page HTML décrivant la ressource. Le code HTML engendré peut contenir des liens hypertextes sur les autres ressources reliées à la ressource courante. Nous obtenons ainsi un navigateur hypertexte sur un graphe RDF distant (celui de DBpedia). Cette technologie permet donc d'engendrer un navigateur hypertexte sur un SPARQL endpoint. On peut aussi coupler l'interrogation d'un graphe local avec un SPARQL endpoint distant et réaliser ainsi un mashup de données liées.

\subsection{Contexte de transformation}

Nous définissons la notion de contexte de transformation qui permet au serveur de transmettre au moteur de transformation STTL des informations relatives au contexte d'exécution de la transformation. Par exemple, pour engendrer des liens hypertextes, le moteur de transformation peut avoir besoin du nom du service. La spécification d'un contexte de transformation évite de "coder en dur" de telles informations dans la transformation, de manière à la rendre le plus générique possible. 
Le contexte peut être consulté par le moteur de transformation au moyen d'une fonction d'extension SPARQL st:get. Les paramètres possibles du contexte sont les suivants : le nom du service, le nom du profil, le nom de la transformation, l'URI de la ressource courante. Voici un exemple de contexte :

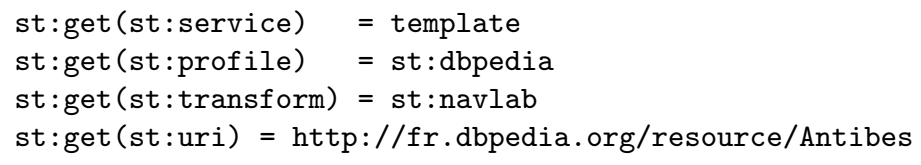

Le contexte peut être utilisé par la transformation pour stocker des informations au moyen de la fonction st: set :

st: set (st:mode, "debug")

Nous généralisons le contexte en permettant aux utilisateurs de spécifier des paramètres supplémentaires dans un profil, paramètres qui sont transmis au contexte de la transformation. Pour cela nous introduisons une propriété st:param dans le profil. La valeur de cette propriété est un nœud vide qui possède un ensemble de propriétés qui représentent les paramètres supplémentaires à transmettre au contexte. L'exemple suivant définit une propriété st: lang qui permet de spécifier la langue de la transformation. La transformation peut alors consulter la valeur de ce paramètre au moyen de l'appel de fonction st:get (st:lang).

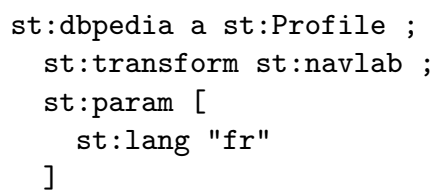

Le contexte peut ainsi être utilisé pour paramétrer des informations relatives au format de présentation, par exemple HTML et CSS. Il peut être utilisé pour spécifier le comportement initial d'objets graphiques tels que des cartes : zoom, taille de la carte, icône de marqueur sur la carte, etc.

Des propriétés relative au Linked Open Data peuvent être définies. Nous avons par exemple introduit une propriété st: lodprofile qui permet de spécifier le profil à utiliser pour un espace de nommage particulier. Par exemple, une ressource appartenant à l'espace de nommage de DBpedia http://fr.dbpedia.fr/resource peut être traitée par le profil spécifique du navigateur DBpedia :

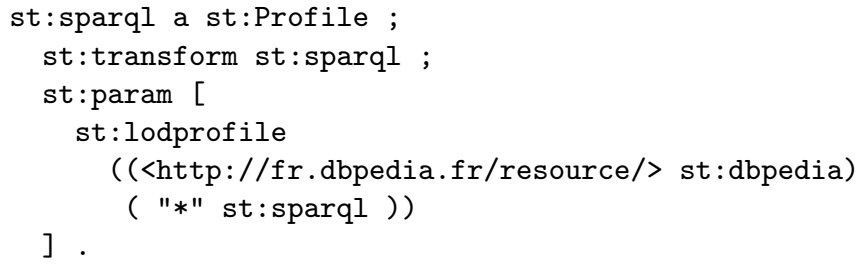

Différents types de ressources peuvent ainsi être traités avec des profils appropriés. 


\subsection{Liens hypertextes dynamiques}

Une des clés de notre approche et du système développé réside en la capacité à engendrer dynamiquement des liens hypertextes dans le code HTML produit. Lorsque l'un de ces liens est suivi, un nouvel appel au serveur est produit, pour engendrer de nouvelles pages HTML relatives à une nouvelle ressource (avec de nouveaux liens hypertextes), grâce à une nouvelle transformation RDF2HTML.

Voici un exemple d'un tel lien hypertexte; l'attribut href de l'élément a a pour valeur un URL qui contient une requête au service STTL du serveur :

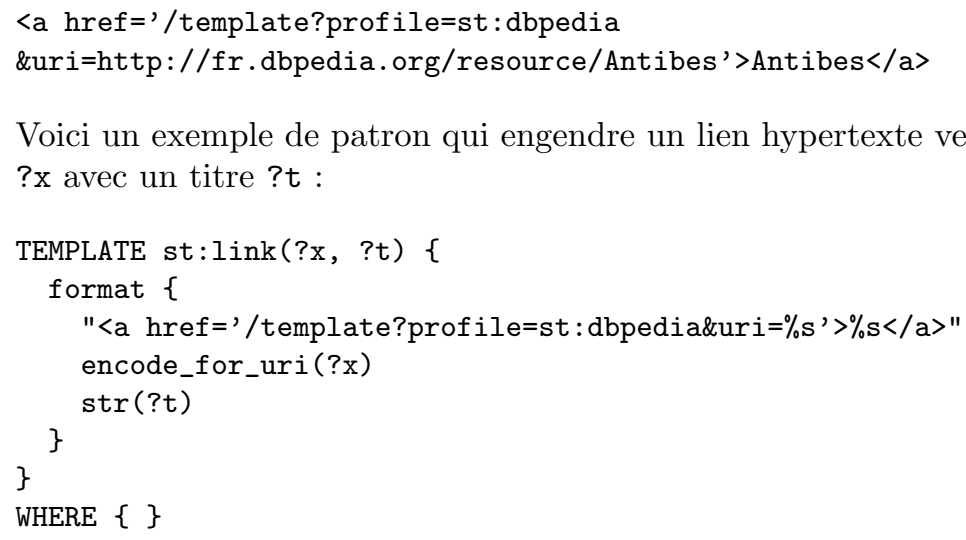
?x avec un titre ?t :

Voici un exemple de patron qui engendre un lien hypertexte vers une ressource

Les information relatives au serveur peuvent être représentées dans le contexte :

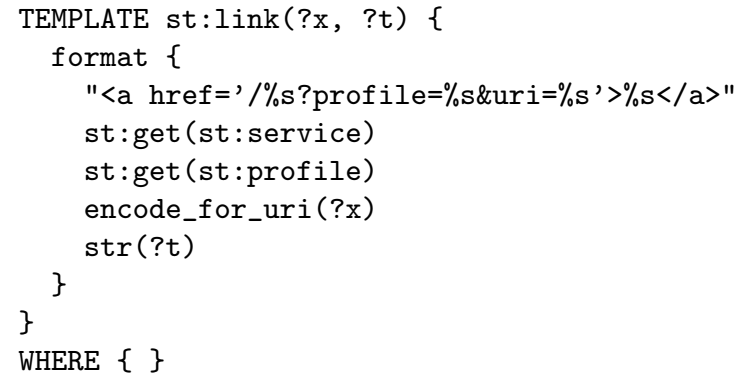

\section{Trois navigateurs ALIGATOR}

Plusieurs exemples de navigateurs construits avec ALIGATOR en réponse à différents besoins sont disponibles en ligne sur un serveur de démonstration ${ }^{7}$. Nous présentons ici quatre d'entre eux. Le code source est disponible dans la distribution de Corese-KGRAM ${ }^{8}$ [2] [6].

\footnotetext{
${ }^{7}$ http://corese.inria.fr

${ }^{8} \mathrm{http}: / /$ wimmics.inria.fr/corese
} 


\subsection{Navigateur pour un service SPARQL}

Nous avons conçu un navigateur ALIGATOR permettant d'exécuter une requête SPARQL et d'en présenter le résultat en HTML. Le résultat d'une requête de la forme SELECT ou ASK est traduit en RDF en utilisant le vocabulaire W3C RDF Data Access Working Group ${ }^{9}$. Une transformation RDF2HTML est ensuite appliquée sur ce graphe. Nous avons défini le profil st:sparql pour identifier cette transformation. Pour les requêtes de la forme CONSTRUCT ou DESCRIBE, la transformation st: sparql est directement appliquée sur le graphe RDF résultat. Les règles de cette transformation sont disponibles en ligne ${ }^{10}$.

La figure 2 est une capture d'écran de la transformation du profil st:sparql appliquée au résultat d'une requête SPARQL (cela est visible dans l'URI entrée dans le navigateur Chrome utilisé). Les liens hypertextes visibles sur la page HTML générée sont des liens vers le serveur ALIGATOR comme expliqué précédemment.

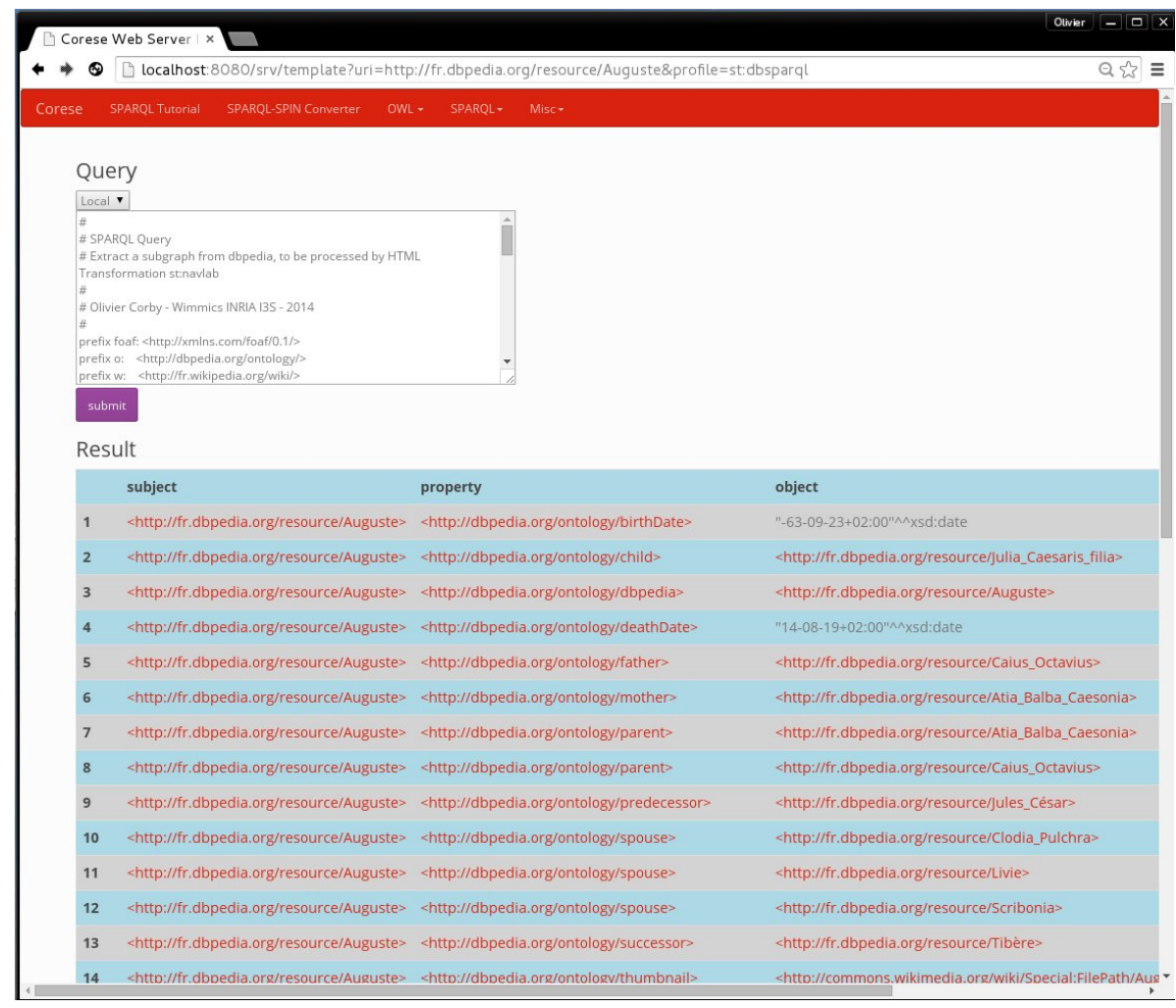

Figure 2: Navigation dans les résultats d'une requête SPARQL

Considérons par exemple la requête SPARQL suivante : SELECT ?x ?n WHERE ?x rdfs:label ?n et l'exemple suivant de solution à cette requête, exprimée en

\footnotetext{
${ }^{9}$ http://www.w3.org/2001/sw/DataAccess/tests/result-set.n3

${ }^{10} \mathrm{http}: / /$ ns.inria.fr/sparql-template/
} 
$\mathrm{RDF}:$

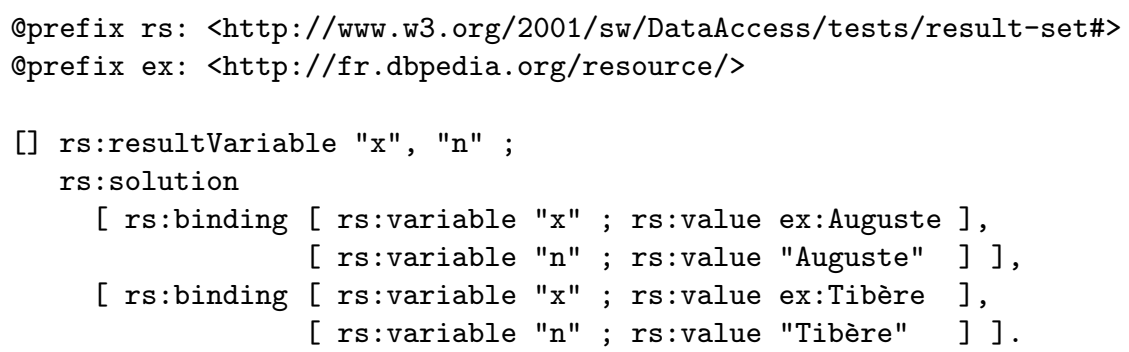

Voici le patron principal de la transformation st:sparql qui traite les résultats des requêtes de la forme SELECT :

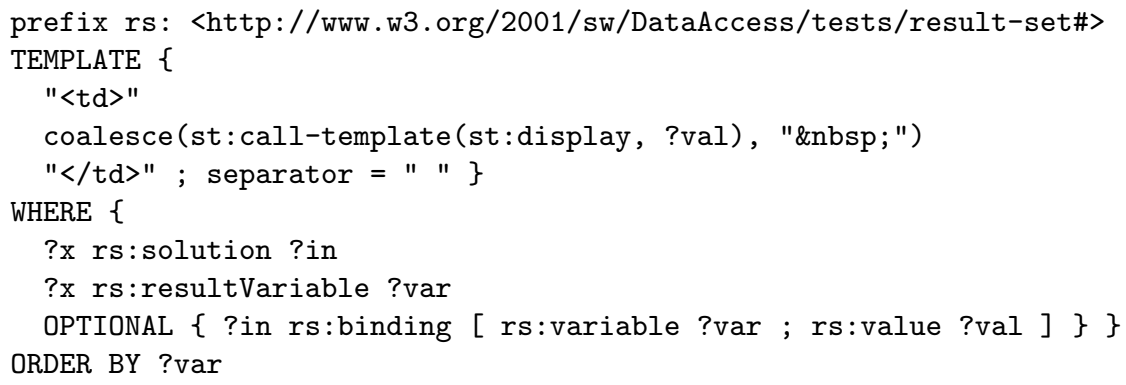

La clause WHERE de ce patron se focalise sur une solution ?in qui est un ensemble de liaisons de variables. La clause optionAL énumère ces liaisons de variables ; cette énumération est dans un sous-graphe optionnel car il se peut que certaines variables (?val) n'aient pas de valeur. La clause TEMPLATE engendre une cellule de table HTML pour chaque variable (?val) avec le résultat de la présentation de la valeur st:call-template(st:display, ?val), ou bien un espace s'il n'y a pas de valeur disponible.

\subsection{Navigateur DBpedia}

Avec la même technologie, il est également possible de concevoir des navigateurs dédiés à des domaines ou des applications spécifiques. Nous avons ainsi développé un navigateur hypertexte dédié à certaines ressources de DBpedia : les personnes et les lieux. Il repose sur un serveur ALIGATOR offrant un service de transformation STTL avec une nouvelle transformation dédiée, dans le profil st:navlab. Son principe de fonctionnement est le suivant. Une requête SPARQL de la forme CONSTRUCT interroge le graphe distant DBpedia, avec une clause SERVICE, sur une personne ou un lieu et retourne un graphe RDF résultat. La transformation st:navlab est ensuite appliquée sur ce graphe résultat. Elle engendre une page HTML dédiée à la présentation des ressources retournées par la requête SPARQL. Les lieux sont géolocalisés sur une carte interactive. La figure 3 est une capture d'écran d'une page HTML générée par le navigateur ALIGATOR pour DBpedia. L'URI entrée dans le navigateur Chrome 
utilisé est un exemple de requête HTTP envoyée au serveur ALIGATOR. Celuici produit en réponse une page HTML engendrée dynamiquement avec le profil st:dbpedia qui utilise la transformation st:navlab.

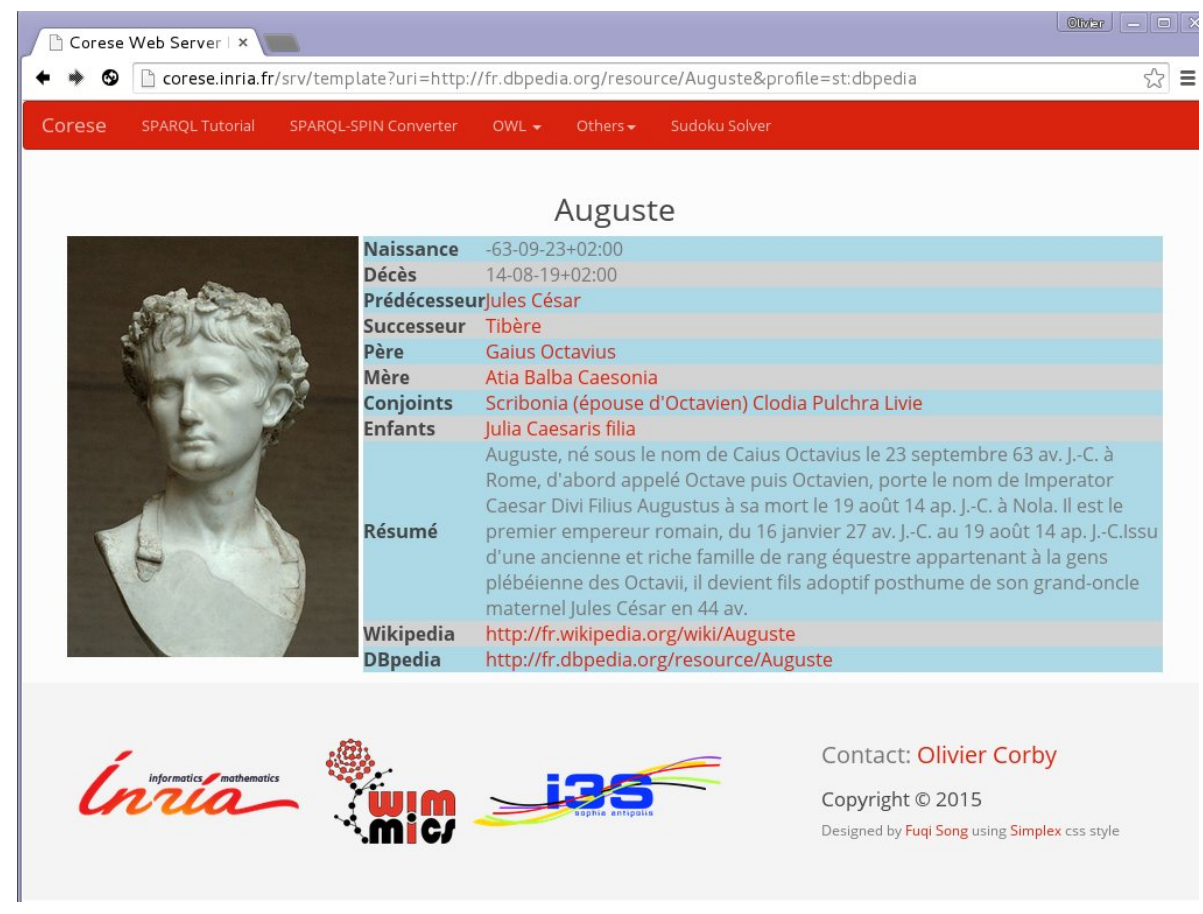

Figure 3: Navigateur DBpedia

\subsection{Navigateur Historique}

Nous avons développé un troisième type de navigateur qui permet de présenter les données issues d'un graphe local lié à un graphe distant, selon le principe du Web de données liées. La source distante est encore une fois DBpedia ; le graphe RDF local contient un ensemble d'événements et de personnages historiques dont les URI sont ceux de DBpedia ${ }^{11}$. Les énoncés RDF locaux sont stockés dans des graphes nommés annotés avec des thèmes tels que la France, l'Empire, etc.

La transformation RDF2HTML que nous avons développée engendre une page HTML par siècle. Dans chaque siècle, les ressources sont classées par ordre chronologique et rangées dans les colonnes d'une table en fonction du thème de leur graphe nommé. Par exemple, une colonne "France" pour les descriptions dans le graphe annoté par le thème "France". Des liens hypertextes vers les ressources correspondantes de DBpedia sont engendrés selon le même

${ }^{11}$ http://fr.dbpedia.org/resource/ 


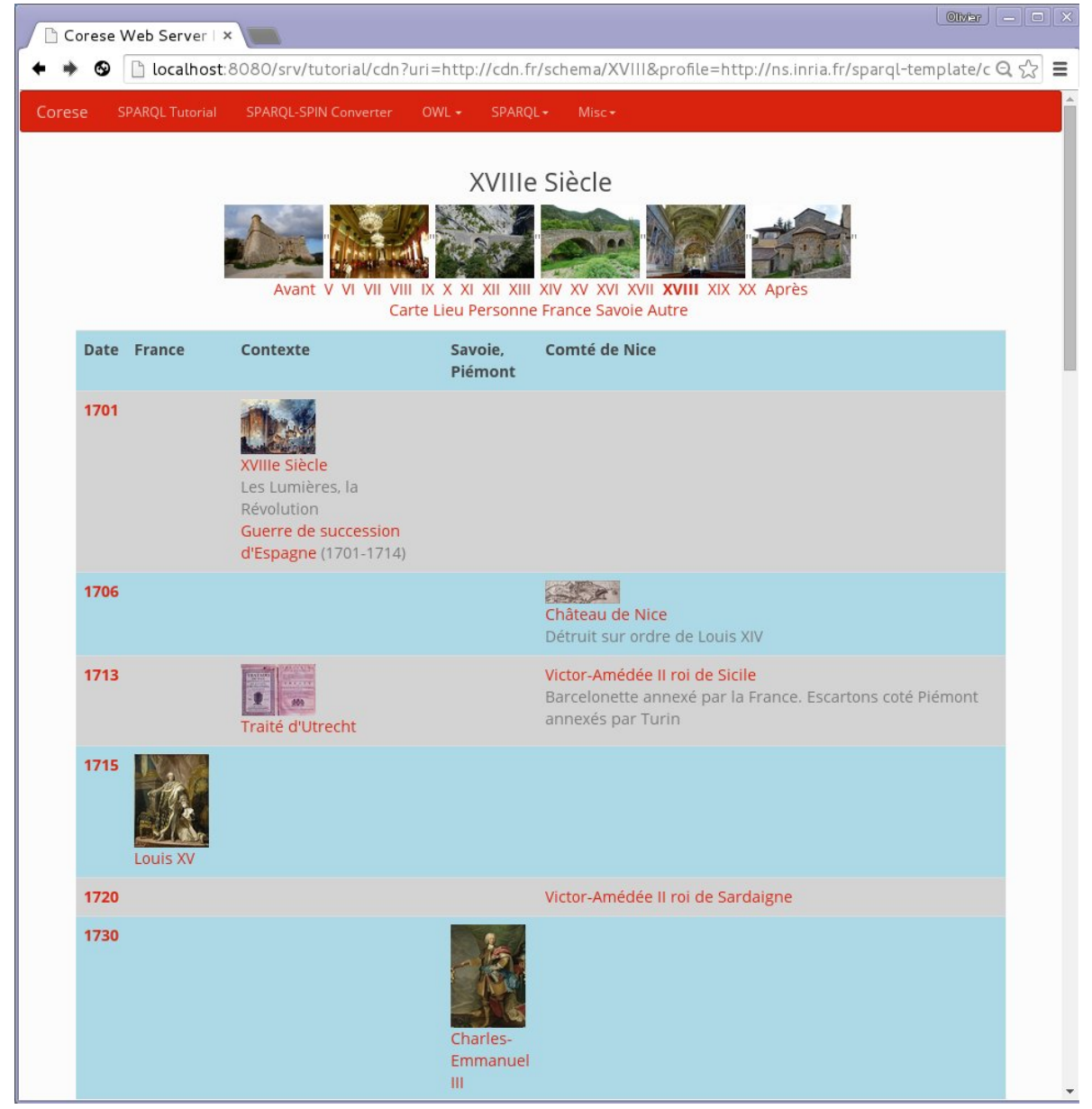

Figure 4: Navigateur historique 
principe décrit dans la section précédente (avec la transformation st:navlab). La figure 4 est une copie d'écran d'une page HTML engendrée par ce navigateur historique.

Le patron suivant joue un rôle essentiel dans la transformation st: navlab. Sa clause WHERE retourne les dates (?d) comprises dans un intervalle (par exemple un siècle), triées par ordre chronologique. Sa clause TEMPLATE permet d'engendrer une ligne de table HTML pour chaque date et une cellule de table pour chaque thème dans laquelle sont affichées les éventuelles ressources correspondant à la date et au thème.

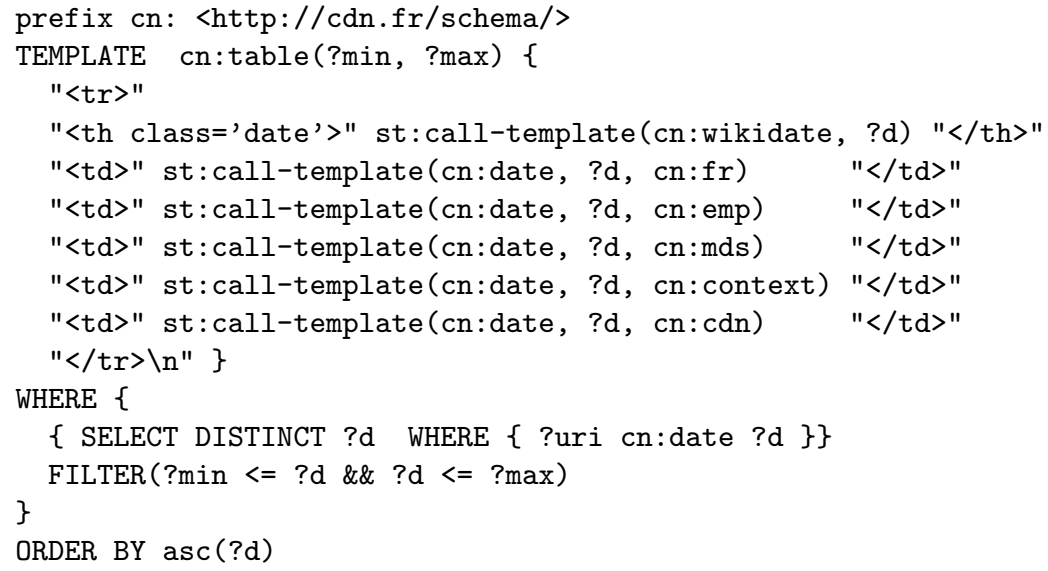

\subsection{Navigateur sur une base de requêtes SPARQL}

Nous avons écrit une transformation STTL st:web permettant de gérer une base de requêtes SPARQL prédéfinies. Celles-ci sont stockées dans un fichier $\mathrm{RDF}$ et sont gérées sous forme d'un graphe RDF qui peut ainsi être l'objet d'une transformation. Le patron suivant est central dans la transformation. Il récupère les requêtes prédéfinies et engendre un sélecteur HTML avec les noms des requêtes (valeurs des propriétés st: name).

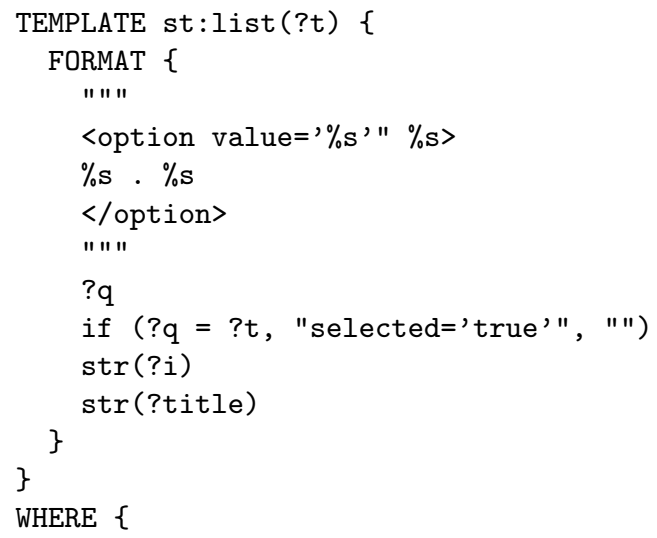




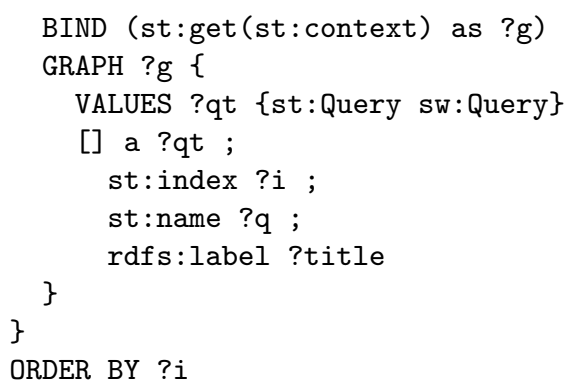

Nous avons développé deux applications à partir de cette transformation générique. La première application est un tutoriel sur le langage SPARQL avec 56 requêtes prédéfinies ${ }^{12}$ et des liens hypertextes vers la recommandation W3C. La transformation permet d'afficher à l'utilisateur une liste de requêtes SPARQL prédéfinies. L'utilisateur peut en sélectionner une qui s'affiche alors dans un champ d'édition dédié; il peut éventuellement en modifier le texte avant de la soumettre au serveur SPARQL. La requête est alors exécutée sur le graphe RDF de l'application. Le résultat de la requête retourné par le serveur est présenté dans un format HTML calculé par une deuxième transformation STTL. Ce format contient des liens hypertextes et permet ainsi de naviguer dans le graphe RDF de l'application. Le résultat de la requête est affiché sous le texte de la requête soumise; il est ainsi possible d'analyser le résultat au vu de la requête qui est elle-même toujours éditable. Cela permet à l'utilisateur de progresser dans son apprentissage du langage SPARQL en modifiant pas à pas sa requête et en constatant l'effet de ses modifications sur les résultats retournés par le serveur SPARQL. La figure 6 montre une copie d'écran du tutoriel. Cette application est utilisée dans le MOOC Inria-uTOP "Web sémantique et web de données" ${ }^{13}$. Le tutoriel propose des exercices à l'aide d'un énoncé, d'un patron de requête SPARQL à compléter et la requête solution. Un lien hypertexte permet d'accéder à la solution de l'exercice, un autre lien permet de revenir au patron de requête à compléter et un troisième lien dynamique permet de soumettre la requête.

La seconde application [de la transformation STTL permettant de gérer des requêtes SPARQL] est un navigateur Linked Data pour visualiser des données RDF extraites des textes du rapport d'activité d'Inria. Ce navigateur a été développé dans le cadre d'un projet Inria interne. La transformation permet de gérer un ensemble de requêtes prédéfinies sur les équipes de recherche, les thématiques de recherche et les coopérations nationales ou internationales. Les requêtes sont résolues sur les données extraites du rapport d'activité, des bases internes de description des équipes et de DBpedia par exemple pour savoir que Manchester est en Angleterre. Les ressources géographiques issues de DBpedia sont géolocalisées sur la base de leur $\mathrm{URI}^{14}$. La figure ?? montre une copie d'écran de l'application.

\footnotetext{
${ }^{12} \mathrm{http}: / /$ corese.inria.fr/data/tutorial/workflow.ttl

13 http://corese.inria.fr/srv/tutorial/rdf

${ }^{14}$ http://fr.dbpedia.org/resource
} 


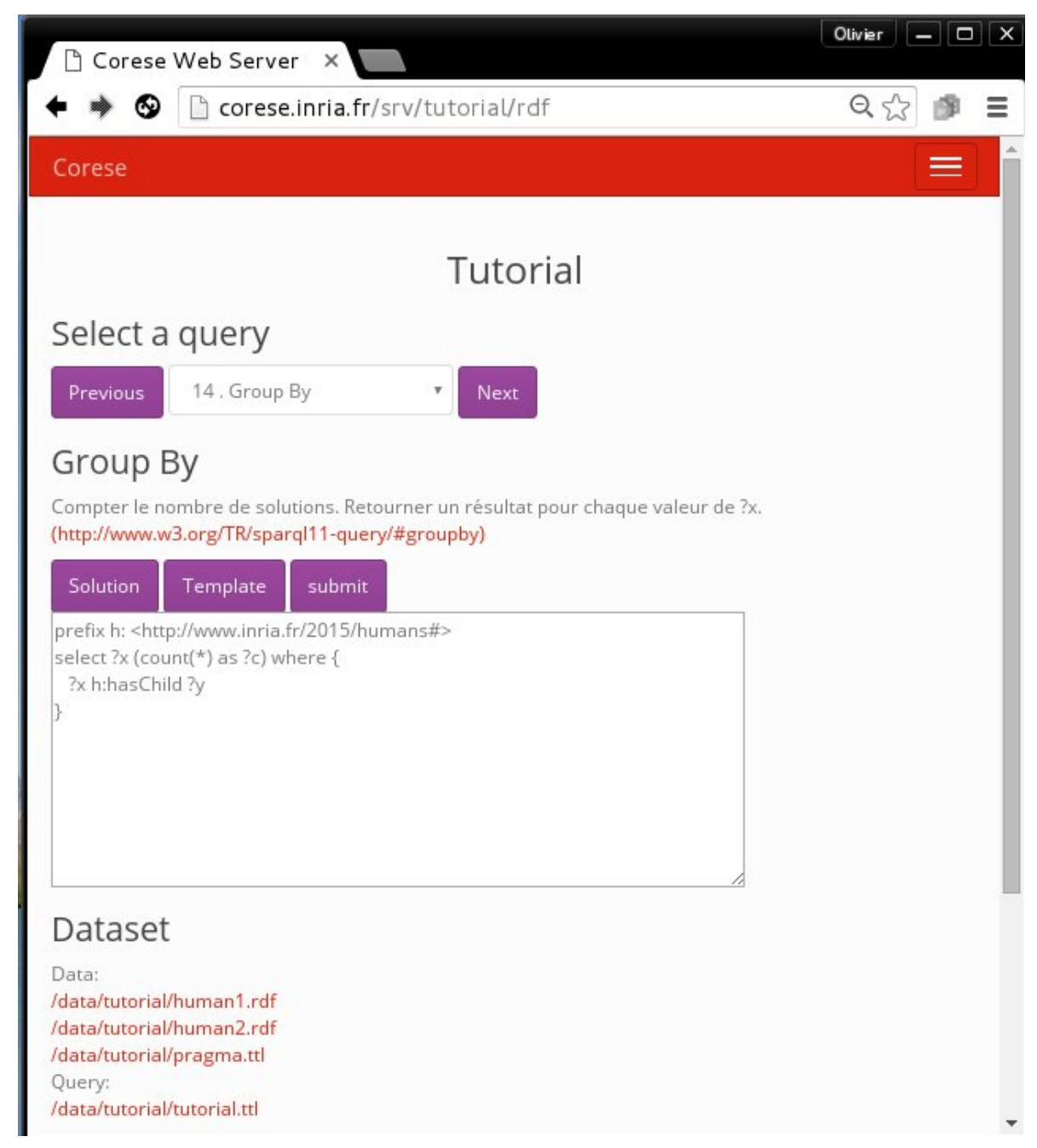

Figure 5: Tutoriel SPARQL 


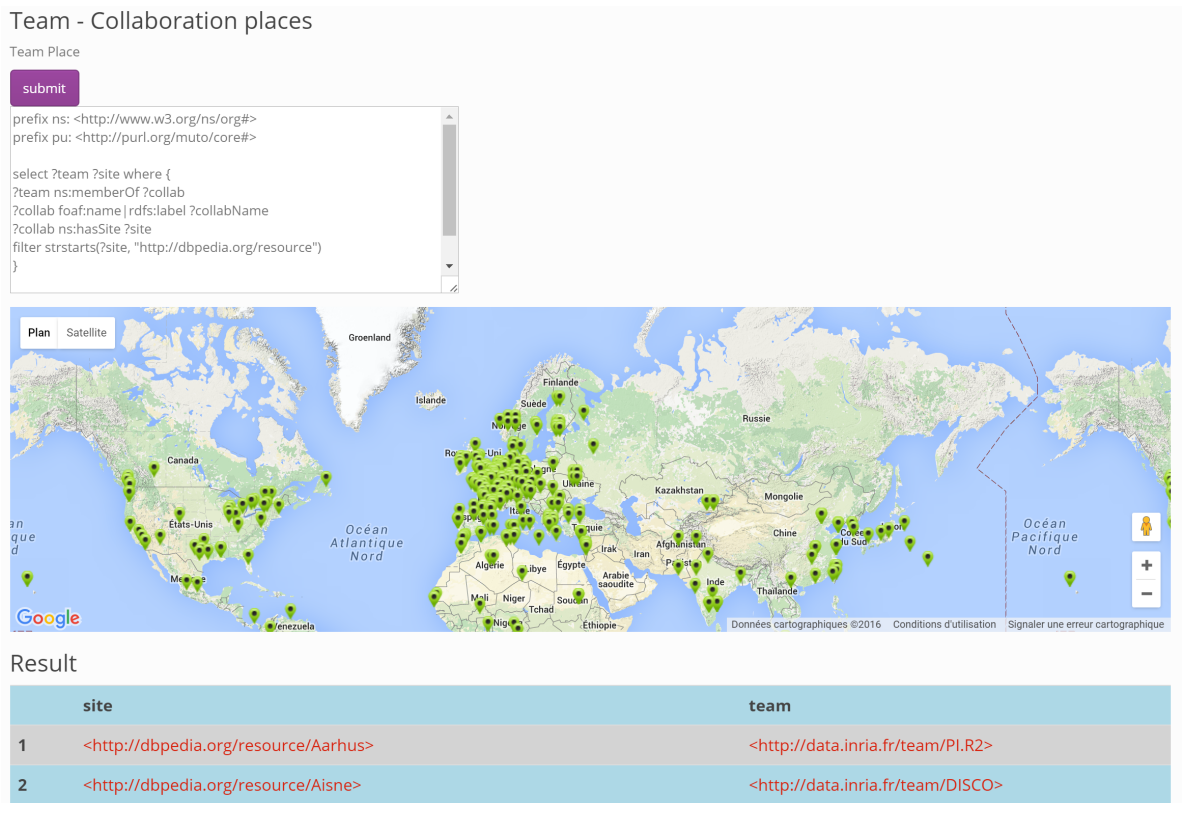

Figure 6: Navigateur sur le rapport d'activité d'Inria

\section{Conclusion}

Nous avons présenté la plate-forme ALIGATOR permettant de concevoir des navigateurs pour les données liées du Web sémantique. Elle repose sur le langage STTL qui est une extension de SPARQL permettant d'écrire des transformations déclaratives de RDF vers des formats textuels, en particulier ici vers HTML. Le moteur de transformation STTL et le serveur STTL qui constituent le navigateur sont disponibles (en open source) dans la plate-forme Corese. Plusieurs applications de cette technologie sont disponibles en ligne sur un serveur de démonstration à l'adresse http://corese.inria.fr.

Un avantage de cette approche, d'un point de vue technique, est qu'elle ne nécessite pas d'apprendre un nouveau framework Web. Il suffit de connaître SPARQL et HTML (et éventuellement JavaScript). STTL permet ainsi de passer "directement" de RDF à HTML avec SPARQL, sans autre langage de programmation.

En termes d'ingénierie des connaissances, les avantages de cette approche sont multiples. Tout d'abord, le fait de reposer sur le langage SPARQL est un atout du point de vue de la réutilisation possible de connaissances expertes : avec une nouvelle forme de requête dont la clause WHERE est commune aux autres formes de requêtes SPARQL, STTL permet d'envisager de réutiliser des patrons de toutes formes de requêtes lorsque ceux-ci ont été capitalisés. Egalement, comme pour l'écriture de requêtes SPARQL en général, les patrons de conception mis en œuvre dans la construction des bases RDF sur lesquelles opèrera 
une transformation STTL peuvent être réutilisés pour écrire les patrons qui composent celle-ci. Enfin, la déclarativité du langage STTL permet de capturer dans les clauses TEMPLATE les connaissances expertes nécessaires pour opérer des transformations sur des données RDF. Les transformations STTL peuvent être vues comme des connaissances de présentation capitalisables, partageables, réutilisables dans des scénarios ou selon des points de vue sur les données similaires.

Dans la continuité de ces conclusions, nous envisageons d'une part d'exploiter les fonctions de HTML 5 couplées avec JavaScript ainsi que d'engendrer des vues graphiques avec des librairie dédiées (e.g. 3D.js). Nous projetons d'autre part d'explorer plus avant la capitalisation de patrons de requêtes SPARQL et patrons de présentation HTML et le couplage de ces deux types de patrons.

\section{Acknowledgement}

Nous remercions Eric Toguem (U. de Yaoundé, Cameroun) et Alban Gaignard (CNRS) pour la première version du serveur HTTP qui embarque Corese ainsi que Fuqi Song pour le déploiement du serveur de démonstration corese.inria.fr

\section{References}

[1] Christian Bizer, Ryan Lee, and Emmanuel Pietriga. Fresnel - A BrowserIndependent Presentation Vocabulary for RDF. In Second International Workshop on Interaction Design and the SemanticWeb@ISWC'05, Galway, Ireland, November 2005.

[2] Olivier Corby and Catherine Faron-Zucker. The KGRAM Abstract Machine for Knowledge Graph Querying. In IEEE/WIC/ACM International Conference on Web Intelligence, Toronto, Canada, September 2010.

[3] Olivier Corby and Catherine Faron-Zucker. SPARQL Template : un langage de Pretty-Printing pour RDF. In Proc. 25e Journées francophones d'Ingénierie des Connaissances, Clermont-Ferrand, May 2014.

[4] Olivier Corby and Catherine Faron-Zucker. FunSPARQL: Extension of SPARQL with a Functional Language. Research Report RR-8814, INRIA, December 2015. http://hal.inria.fr/hal-01236947.

[5] Olivier Corby and Catherine Faron-Zucker. STTL: A SPARQL-based Transformation Language for RDF. In Proc. 11th International Conference on Web Information Systems and Technologies, WEBIST 2015, Lisbon, Portugal, May 2015.

[6] Olivier Corby, Alban Gaignard, Catherine Faron-Zucker, and Johan Montagnat. KGRAM Versatile Data Graphs Querying and Inference Engine. In Proc. IEEE/WIC/ACM International Conference on Web Intelligence, Macau, China, December 2012. 
[7] Steve Harris and Andy Seaborne. SPARQL 1.1 Query Language. Recommendation, W3C, 2013. http://www.w3.org/TR/sparql11-query/. 